\title{
Examining doctor-patient communication skills among senior medical students based on calgary cambridge observation guide
}

\author{
Merina Eskandari ${ }^{1 *} \mathbb{D}$, Fakhrolsadat Hosseini ${ }^{2}$, Katayoon Razjouyan $^{3}$, Alireza Abadi ${ }^{4}$ \\ Received: 16 May 2020 \\ Published: 22 Sep 2021
}

\section{Abstract}

Background: This study aimed to determine the level of communication skills in residents of Shahid Beheshti University of Medical Sciences in the final year of the main courses in accordance with the Calgary Cambridge Observation Guide for the purpose of improving their skills and improving the quality and correcting the existing problems.

Methods: In this cross-sectional study, 190 residents of 14 majors were evaluated using a checklist of patient and physician communication skills based on the Calgary Cambridge Guide that has been localized in Persian language and culture using the crosscultural adaptation standard in 6 stages. Content validity was verified by the primary author. The skills within the 71-item checklist were examined via a 3-point Likert-type rating scale, with scores ranging from 1 to 3 (good to poor) and the data were analyzed using the SPSS 16 software.

Results: Of the190 residents, 161 were enrolled in the study. Of them 74 were internal and 87 surgical residents, and 89 were male and 72 were female. The mean score of the total communication skills in 14 major courses was $128.68 \pm 37.2264$; it was $121.7 \pm 36.990$ in the Department of Surgery, and 136.8 \pm 36.073 in the Department of Internal Medicine, with $\mathrm{p}=0.010$, in female students 126.6 and in male student 130.3 with $\mathrm{p}=0.500$.

Conclusion: The score of 71 communication skills points in the list was prepared using the Likert scale option 3. In the overall skills, the weak score is 71 to 118.33 , the average score is 118.34 to 165.66 , and the good score is 165.67 to 213 . Based on the findings of the study and examining the educational curriculum, it can be stated that unlike in psychiatry with an average score of 168.83 , which is at a good level, the other fields are not well-developed and overall the communication skills in the residents, with a mean score of 128.68, are moderately poor and therefore further education and training is needed in these fields for resident students.

Keywords: Calgary Cambridge Guide, Physician-Patient Relations, Communication Skills

Conflicts of Interest: None declared

Funding: None

\section{*This work has been published under CC BY-NC-SA 1.0 license.}

Copyright $\odot$ Iran University of Medical Sciences

Cite this article as: Eskandari M, Hosseini F, Razjouyan K, Abadi A. Examining doctor-patient communication skills among senior medical students based on calgary cambridge observation guide. Med J Islam Repub Iran. 2021 (22 Sep);35:122. https://doi.org/10.471 76/mjiri.35.122

\section{Introduction}

Communication skills with patients is in the heart of

Corresponding author: Merina Eskandari, eskandari@nasrme.ac.ir

1. National Center for Strategic Research in Medical Education, Tehran, Iran

2. School of Medical Sciences, Shahid Beheshti University of Medical Sciences, Tehran, Iran

3. Shahid Beheshty University of Medical Sciences, Tehran, Iran

4. Social Determinants of Health Research Center, Shahid Beheshti University of Medical Sciences, Tehran, Iran medicine and it is also considered as the center of all clin-

$\uparrow$ What is "already known" in this topic:

Globally and according to previous studies, unsuccessful physician-patient communication has been commonly observed, especially in interview methods and patient-resident relationships, and it has been proven that more interactions and further communication skills training to be highly important.

\section{$\rightarrow$ What this article adds:}

this study aimed to examine doctor-patient communication skills among senior residents of Shahid Beheshti University of Medical Sciences based on Calgary Cambridge Observation Guide in 5 specific areas. This study was done to improve the communication skills of senior residents, improve the quality of doctor-patient communication, and correct the existing problems. 
ical measures so that $60 \%$ to $80 \%$ of medical diagnoses as well as a similar proportion of treatment decisions are taken based o $\mathrm{n}$ information obtained from medical interviews (1). Communication skills have attracted much attention in research studies on health care and they are correspondingly recognized as one of the main components of clinical competencies contributing to correct diagnosis and patient compliance with treatment measures. Meanwhile, it has been proved that good communication skills can meet patient satisfaction and their compliance with treatment orders and ultimately improve delivery of health care services (2-4). Lack of communication skills, ie, ineffective communication, can disguise patients' problems and needs in this domain and may result in defects in patient satisfaction (5).

Globally and according to the studies, failed doctorpatient communication has been commonly observed, especially within interview methods, patient-resident relationships as well as positive impacts of education to fix such shortcomings (6-8). According to the investigations by Kurtz and Silverman, doctors lacked training in terms of general communication skills because such skills are mainly acquired, ie, they can be learned but individual clinical experience can play a small role in their improvement. Thus, there is a need to have trainings in this domain which have been performed from 1998 according to Calgary Cambridge Observation Guide (9). An overview of the course units of different levels of medical studies have similarly shown that such skills have not been included in medical education curriculum and doctors' learning process in Iran has been on the basis of indirect and experimental modeling from their teachers prevailed with biomedical attitudes $(10,11)$. Studies in Iran also considered reviews, more interactions, and further communication skills training to be highly important (12-14). Given the number of studies about teaching such skills in Iran and its short history as well as the lack of no compulsory, purposeful, and coherent curriculum on communication skills within universities for residents and the lack of basic appropriate information about the amount of communication skills among residents, examining doctorpatient communication skills among the senior residents of Shahid Beheshti University of Medical Sciences based on Calgary Cambridge Observation Guide was done in 5 specific areas. This was done to improve the senior resident's skills, improve the quality of doctor-patient communication, and correct the existing problems.

\section{Methods}

In this cross-sectional descriptive study, the statistical population consisted of 190 senior residents enrolled in 14 specialties at Shahid Beheshti University of Medical Sciences, including neurosurgery, neurology, dermatology, psychiatry, ophthalmology, infectious and tropical diseases, ENT and head and neck surgery, urology, orthopedics, cardiology, general surgery, obstetrics and gynecology, emergency medicine, and internal medicine. Of the participants, 161 entered in the study because of their presence period, including 74 students in the Department of Internal Medicine and 87 students in the Department of
Surgery. Moreover, gender was considered as another variable in this study (men $(n=89)$; women, $(n=72))$.

The data collection instrument was an observation checklist for doctor-patient communication skills based on Calgary Cambridge Observation Guide translated and revised in line with the Persian culture and language, whose reliability and validity had been already confirmed (15).

Reviewing different research studies in this domain showed that such investigations conducted based on Calgary Cambridge Observation Guide had not localized and translated the given checklist properly according to the Persian culture and language. In fact, they had not appropriately covered Calgary Cambridge Observation Guide. Thus, at first, localization was performed in this study based on the standard cross-cultural adaptation (CCA) (16).

For this purpose, 5 steps were taken after obtaining permission from the main author to translate and employ the checklist in this study: (1) The original text was translated into Persian by 2 individuals, one of them was aware of the problem and the purpose of the study and the other had no knowledge in this regard; (2) Both translations were combined, their differences were resolved, and a final checklist was obtained; (3) The final text was retranslated into its original language by 2 English native speakers; (4) The translations were checked by a committee of authorized experts in the domain of communication skills and the main author, their approvals were acquired, and the final checklist was obtained; and (5) A pilot test was conducted on $10 \%$ of the study samples by the final checklist to enhance its accuracy and confirm its validity. Meanwhile, the checklist included 71 items in 5 separate sections, including skills in the domain of initiating the session, gathering information, providing structure and building relationships, explanation and planning, and closing the session examining doctor-patient communication skills.

Observation was conducted by the researcher according to the checklist. To collect data after obtaining permission from the ethics committee and the organization (the department and clinical units) and informing them and also ensuring the presence of senior residents in hospitals, the researcher attended the given setting several times and evaluated students while they were performing clinical work through direct observations to the extent that residents got accustomed to the presence of the observer and their work was not assumed contrived. After that, the checklists were completed.

The skills within the 71-item checklist were examined via a 3-point Likert-type rating scale, ranging from 1 to 3 (from good to poor), and finally the data were analyzed using the SPSS 16 software. The amount of the total communication skills in 14 specialties in total and that for each of the 5 subgroups was analyzed and specified using the analysis of variance (ANOVA). The scores were calculated and divided into 5 subgroups: initiating the session, gathering information, providing structure and building relationships, explanation and planning, and closing the session given the number of items in each section. In 
this respect, within interpersonal skills for initiating the session with 7 items, scores 7 to 11.66 were considered poor, scores 11.66 to 16.33 moderate, and scores 16.34 to 21 good. Given that 11 items were related to the skills for gathering information, scores 11 to $18.33,18.34$ to 25.66 , and 25.67 to 33 were considered poor, moderate, and good, respectively. Considering that 14 items were related to skills for providing structure and building relationships, scores 14 to 23.33 were assumed poor, scores 23.34 to 32.66 moderate, and scores 32.67 to 42 good. Given that explanation and planning skills comprised of 35 items, scores 35 to $58.33,58.34$ to 81.66 , and 81.67 to 105 were considered poor, moderate, and good, respectively. Considering that 4 items were related to skills for closing the session, scores 4 to 6.66 were assumed poor, 6.67 to 9.33 moderate, and 9.34 to 12 good. Given the total communication skills with 71 items, scores 71 to $118.33,118.34$ to 165.66 , and 165.67 to 213 were considered poor, moderate, and good, respectively.

\section{Results}

The amount of total communication skills in 14 specialties in total and that for each of the 5 subgroups was analyzed.

The mean score of the total the communication skills was $128.68 \pm 37.2264$, except for the senior residents in psychiatry, with the mean score of $167.83 \pm 27.7374$, who were placed at good and strong level. The senior residents of 5 specialties of neurosurgery, neurology, ophthalmology, orthopedics, and obstetrics and gynecology, with the mean scores of $114.00 \pm 28.4681,116.33 \pm 28.5933$, $110.92 \pm 28.2325,114.67 \pm 32.1257$, and $114.89 \pm 28.4681$, respectively, were at poor levels and other specialties at Shahid Beheshti University of Medical Sciences had been placed at moderate to poor levels (Table 1).

The mean score of the total doctor-patient interpersonal skills for initiating the session was $14.09 \pm 3.4806$, and excluding the senior residents of psychiatry, with the mean score of $17.66 \pm 2.0655$ who were placed at a good and strong level, the senior residents enrolled in 8 specialties of neurosurgery, neurology, ophthalmology, orthopedics, obstetrics and gynecology; ear, nose, and throat (ENT) and head and neck surgery, infectious and tropical diseases, and urology were placed at moderate to poor lev- els, respectively, with the mean scores of $12.50 \pm 4.1352$, $13.33 \pm 4.32 .4,13.15 \pm 3.1582,12.58 \pm 3.0883,12.26 \pm 2.1818$, $13.77 \pm 4.1766,13.25 \pm 3.1509$, and $13.30 \pm 3.5292$. Residents had a significant mean difference in the skills at the beginning and at the end of the session; that is, interpersonal skills between the doctor and the patient at the beginning of the session, with $\mathrm{p}=0.02$, and the skill of closing the session, with $p=0.04$. And there is a difference between the disciplines according to their importance and attention to these skills in the residency period and they can be distinguished.

The mean score of the total gathering information skills was $21.85 \pm 6.2020$, and except for the senior residents of psychiatry, with the mean score of $27.83 \pm 3.4880$ who were categorized at a good and strong level, the senior residents in 7 specialties of neurosurgery, neurology, ophthalmology, orthopedics, obstetrics and gynecology, cardiology, and ENT and head and neck surgery with the mean scores of $18.83 \pm 7.2502,19.66 \pm 6.3770,19.23 \pm 4.7461$, $20.41 \pm 5.8536, \quad 19.10 \pm 4.8407, \quad 21.00 \pm 7.3484, \quad$ and $20.25 \pm 6.6062$, respectively, were placed at moderate to poor levels.

The mean score of the total providing structure and building relationship skill was $26.87 \pm 8.169,1$ and excluding the senior residents of psychiatry, with the mean score of $35.16 \pm 4.8339$ who were placed at a good and strong level, the senior residents enrolled in 3 specialties of neurosurgery, neurology, and ophthalmology, with the mean scores of $23.00 \pm 8.9218,23.33 \pm 6.3140$, and $22.84 \pm 7.6458$, respectively, were at poor levels; and 5 specialties of orthopedics, obstetrics and gynecology, cardiology, ENT and head and neck surgery, and infectious and tropical diseases were at moderate to poor levels, with the mean scores of $25.41 \pm 7.5853,24.84 \pm 6.9702,26.57 \pm 8.4826$, $25.44 \pm 8.9178$, and $24.75 \pm 8.7790$ respectively.

The mean score of the total explanation and planning skills was $59.13 \pm 18.9113$, and except for the senior residents of psychiatry with the mean score of $77.16 \pm 17.0460$, who were categorized at a moderate to strong level, the senior residents in 7 specialties of neurosurgery, neurology, ophthalmology, infectious and tropical diseases, ENT and head and neck surgery, orthopedics, and obstetrics and gynecology, with the mean scores of $53.50 \pm 19.3364$, $54.16 \pm 11.9065, \quad 49.92 \pm 13.5613, \quad 55.87 \pm 21.1419$,

\begin{tabular}{|c|c|c|c|c|c|}
\hline Row & Field & Number & Average & Standard deviation & Score range \\
\hline 1 & Neurosurgery & 6 & 114.00 & 41.7995 & $79-191$ \\
\hline 2 & Neurology & 6 & 116.33 & 28.5913 & $79-148$ \\
\hline 3 & Dermatology & 3 & 139.33 & 43.0387 & $113-189$ \\
\hline 4 & Psychiatry & 6 & 167.83 & 27.7374 & $133-211$ \\
\hline 5 & Ophthalmology & 13 & 110.92 & 28.2325 & $79-165$ \\
\hline 6 & Infectious and tropical diseases & 8 & 120.12 & 41.2810 & $79-192$ \\
\hline 7 & ENT and head and neck surgery & 9 & 124.58 & 40.3426 & $79-186$ \\
\hline 8 & Urology & 10 & 137.50 & 41.7219 & $79-194$ \\
\hline 9 & Orthopedics & 12 & 114.67 & 32.1257 & $79-179$ \\
\hline 10 & Cardiology & 14 & 132.21 & 34.6924 & 79-189 \\
\hline 11 & General surgery & 17 & 137.24 & 45.3548 & $79-228$ \\
\hline 12 & Obstetrics and gynecology & 19 & 114.89 & 28.4681 & $79-180$ \\
\hline 13 & Emergency medicine & 16 & 138.31 & 34.4552 & $95-199$ \\
\hline 14 & Internal medicine & 22 & 138.86 & 38.0179 & $79-187$ \\
\hline 15 & Total & 161 & 128.68 & 37.2264 & $79-228$ \\
\hline
\end{tabular}


$58.33 \pm 19.3971,50.83 \pm 15.7412$, and $52.63 \pm 14.6598$, respectively, were placed at poor levels. The mean scores of other specialties were at moderate to poor levels.

The mean score of the total closing the session skill was $6.72 \pm 2.5179$, and excluding the senior residents of psychiatry, with the mean score of $10.00 \pm 2.0976$ placed at a strong level, the senior residents enrolled in 7 majors of neurosurgery, neurology, ophthalmology, infectious and tropical diseases, ENT and head and neck surgery, orthopedics, and obstetrics and gynecology were at poor levels, with the mean scores of $6.16 \pm 2.5625,5.83 \pm 1.6020$, $5.76 \pm 2.4205,6.00 \pm 2.5634,6.00 \pm 1.8708, \quad 5.41 \pm 2.0652$, and $6.05 \pm 2.2473$, respectively. The mean scores of other specialties were at moderate to poor levels. Residents of this skill with $\mathrm{p}=0.040$ ha significant mean differences.

The amount of the total communication skills in 14 specialties and that for each of the 5 subgroups in the residents of the Department of Internal Medicine and the Department of Surgery and in the male and female residents were analyzed and specified using t test.

The mean score of the total skills in the Department of Surgery was $121.7 \pm 36.990$ and it was $136.8 \pm 36.073$ in the Department of Internal Medicine with $\mathrm{p}=0.010$, but for each of the 5 subgroups, the scores of residents in the Department of Internal Medicine were higher than those obtained by surgery residents. Separately, the residents of the Department of Internal Medicine were at moderate levels and the residents of the Department of Surgery were placed at poor levels in terms of explanation and planning skills as well as those for closing the session.

The mean score of the total communication skills in female residents was $126.6 \pm 34.718$ and it was $130.3 \pm 39.251$ in male residents with p0500, but for each of the 5 subgroups the scores of male residents were higher than those obtained by the female. Separately, the male residents were at moderate levels and female residents at poor levels in terms of explanation and planning skills as well as those for closing the session.

\section{Discussion}

To respond to the overall purpose of the study, it can be stated that the amount of communication skills was not at a good level except for the specialty of psychiatry, with the mean score of 167.83 , which was placed at a good level. Overall, the given skills, with a mean score of 128.68 , were at moderate to poor levels. In this respect, the results of the study by Labbaf et al showed the need for training and frequent feedback in order to improve their communication skills (17). Moreover, Bragard et al conducted a study on teaching communication skills to residents aimed at determining the variables associated with learning skills on 56 residents in the specialties of oncology, obstetrics and gynecology, general surgery, and gastroenterology, which revealed defects in communication skills among residents and the need for teaching these skills to gain work experience and also confirm enhanced support skills in residents (18). Considering communication skills training in medicine as well as needs assessment of the educational status of such skills in Daisho School of Medicine, Suzuki et al conducted an investiga- tion on 117 students, among them, 54 individuals were senior residents. The results of the given study in all cases revealed poor communication skills and raised the need for formal and informal training of such skills (19). Nevertheless, the study by Nonezhad et al on communication skills among the residents of internal medicine at Shiraz University of Medical Sciences showed that the amount of total communication skills were evaluated at a good and acceptable level (11). On the other hand, and according to the review of educational curriculum of different specialties based on their contents on communication skills, it was concluded that in the specialty of psychiatry, unlike other disciplines, teaching and implementing communication skills were completely performed for all mental conditions and age groups (10).

Except for the doctor-patient interpersonal skills for initiating the sessions, with a mean score of 14.09 , the residents were at moderate to poor levels in other 4 subgroups of skills. In this regard, the results of the study by Rahimzadeh et al on the evaluation of interpersonal communication skills among residents of Hazarate Rasoul Complex enrolled in the specialties of obstetrics and gynecology, internal medicine, neurology, neurosurgery, ENT and head and neck surgery, emergency medicine, occupational medicine, anesthesiology, ophthalmology, psychiatry, orthopedics, general surgery, as well as dermatology showed a mean score of $81.23 \# 10.93$ (at the range of 32 to 99) at a moderate level for such skills wherein the residents of occupational medicine and emergency medicine had obtained the highest and the lowest scores, respectively (20). The study by Agrawal evaluating interpersonal communication skills among doctors specialized in internal medicine using the MAAS-Global Rating List also suggested that the mean scores in all domains were poor and lower than satisfactory level (21). On the other hand, Ishikawa focused on information-sharing skills among residents through examining the films of medical interviews of 25 residents at the Tokyo Teaching hospital with Route Analysis System along with coding and concluded that such skills were placed at a poor level. It was also concluded that doctors who had further concentrated on teaching and implementing communication skills were endowed with higher self-confidence and they were also stronger in terms of the skills of giving information to patients as well as explanatory and planning ones (22).

Given the amount of communication skills for each specialty, the findings of this study indicated that the senior residents of 5 specialties of neurosurgery, neurology, ophthalmology, orthopedics, and obstetrics and gynecology with the mean scores of 114.00, 116.33, 110.92, 114.67, and 114.89 , respectively, were placed at a poor level and 7 specialties of dermatology, internal medicine, urology, ENT and head and neck surgery, infectious and tropical diseases, emergency medicine, cardiology, and general surgery with mean scores of 139.33, 138.86, 137.50, $124.56,120.12,138.31,132.21$, and 137.24 , respectively, were placed at moderate to poor levels. In this regard, a study was conducted regarding the evaluation of communication skills among 26 residents in dermatology, internal medicine, urology, ENT and head and neck surgery, neu- 
rology, radiology, and obstetrics and gynecology at Gilan University of Medical Sciences in 2003 using a multiplechoice questionnaire by Heidarzadeh et al and revealed a poor level of communication skills in these specialties with the mean scores of $8.78 \pm 2.6$ (out of 25) (23). The investigation by Porcerelli et al on scores obtained by residents in communication skills using the Kalamazoo Essential Elements Communication Checklist, a good level of communication skills was observed among the residents of internal medicine, dermatology, neurology, and orthopedics (24). Moreover, the study by Khoshrang et al that examined the quality of education provided in the Clinical Skills Center from the attitudes of residents from Gilan University of Medical Sciences revealed the poor level of residents in the specialty of obstetrics and gynecology $(54.5 \%)$ in terms of communication skills compared to other specialties, including internal medicine $(90.8 \%)$, pediatrics $(68.2 \%)$, urology $(100 \%)$, orthopedics (77.3\%), ENT an head and neck surgery $(90.9 \%)$, and general surgery $(68.2 \%)(25)$. Additionally, the study by Zamani et al on the impact of communication skills training on clinical skills among 13 residents of internal medicine and infectious disease at Isfahan University of Medical Sciences between 2003 to 2006 using Calgary Cambridge Observation Guide for training and skills assessment showed increased mean scores of residents in both specialties after teaching communication skills in terms of skills of history-taking from 66 to 81, physical examination skills from 60 to 92 , diagnostic skills from 96 to 100 , management skills from 60 to 90 , and clinical skills from 70 to 87 , suggesting a weakness in their communication skills and the need for teaching these skills in order to enhance clinical skills among residents (26). In this line, Norgaard et al conducted a study on the effect of communication skills training on increased self-efficiency among health care professionals, including 181 residents of orthopedics after participating in a 3-day training course of communication skills before, immediately after, and 6 months after training, and their results revealed augmented mean scores of self-efficacy compared with those obtained by patients from 6.68 to 7.88 and the need for communication skills training in these residents (27).

The residents of the Department of Internal Medicine were endowed with stronger levels of communication skills. In this regard, the study by Ahmed al-Ansari in 2012 revealed that that the residents of internal medicine had higher levels of skills, especially in the domains of explanation and planning skills and those for giving information to patients compared with residents of surgery (28).

The mean scores of male residents were higher than those obtained by the female. However, the results of the study by Nonezhad et al and the investigation by Bilund et al suggested that female residents had acquired higher levels of communication skills than the male $(11,29)$.

\section{Conclusion}

This study shows that considering the current curriculum of the residents, there is a need to emphasize planned education for all residents.

\section{Ackowledgment}

The authors would like to thank all the main authors of the Calgary Cambridge Guide, especially Dr Suzanne M Kurtz.

\section{Conflict of Interests}

The authors declare that they have no competing interests.

\section{References}

1. Lynch DJ, McGrady AV, Nagel RW, Wahl EF. The patient-physician relationship and medical utilization. Prim Care Companion J Clin Psychiatry. 2007;9(4):266-270.

2. Ha JF, Longnecker N. Doctor-patient communication: a review. Ochsner J. 2010;10(1):38-43

3. Corkill C. The doctor patient relationship. In:George IS. Cole's Medical Practice in NewZealand 2011, 10th Edition. New Zealand: Medical Council of New Zealand; 2010:p. 34-40.

4. Farajzadeh S, Noohi E, Mortazavi H. Interns' Communication with Patients during Interview: the Perspectives of Patient and Observer. Iran J Med Educ. 2006;6(2):79-86. [In Persian]

5. Smith CM. Scripts: a tool for cognitive rehearsal. J Contin Educ Nurs. 2011;42(12):535-536.

6. Chang JT, Hays RD, Shekelle PG, MacLean CH, Solomon DH, Reuben DB, et al. Patients' global ratings of their health care are not associated with the technical qualitty of their care. Ann Intern Med. 2006 May 2;144(9):665-72.

7. Dehnavie R, Dehghani MR, Ebrahimipour H, Nouri Hekmat $S$, Merolhasani MH, Soroudi Nasab S, et al. Assessment of Communication Skills in Advisors of Undergraduate Students in Kerman University of Medical Sciences. Stud Develop Med Educ. 2012;8(2):189-94. [In Persian]

8. Simmenroth-Nayda A, Weiss C, Fischer Th, Himmel W. Do communication training programs improve students' communication skills? a follow-up study. BMC Res Notes. 2012;5:486.

9. Sanson-Fisher R, Cockburn J. Effective teaching of communication skills for medical practice: selecting an appropriate clinical context. Med Educ. 1997;31(1):52-7.

10. Rahman A. Initial assessment of communication skills of intern doctors in history -taking. Med Teach. 2000;22(1-2):184-8.

11. Kurtz SM, Silverman JD. The Calgary Cambridge Referenced Observation Guides: an aid to defining the curriculum and organizing the teaching in communication training programmes. Med Educ Commun Sites. 1996;30(2):83-89.

12. Office of Presidency and Public Relations at Shahid Beheshti University of Medical Sciences and Health Services in Tehran. Comprehensive Guide to Education at Shahid Beheshti University of Medical Sciences, 2000-2001 Academic Year.

13. Nonezhad N. Assessment of communication skills of internal residents of Shiraz University of medical science. A Thesis submitted to the school of medicine in partial fulfillment of the requirements for the degree of doctor of medicine 2010. [In Persian]

14. Mirzakhani N, Sheikh Fendreski T, Tabatabae M, Soltani-arabshahi $\mathrm{K}$. A survey on communication skills of undergraduate student of shahid Beheshti University of Medical Sciences. Sci J Rehabil Med. 2014;3(1). [In Persian]

15. Eskandari M, Hosseini F, Razjouyan K, Abadi A. Calgary Cambridge Observer Guide indigenization for measuring physicianpatient communication skills Based on the Cross-Cultural Adaptation Standard in Iran. J Med Cultiv. 2019;28(3):59-70.

16. Beaton D, Bombardier C, Guillemin F, Bosi Ferraz M. Recommendations for the Cross-Cultural Adaptation of the DASH \& Quick DASH Outcome Measures. Institute for Work \& Health 2002, 2007.

17. Labaf A, Jamali K, Jalili M, Baradaran HR, Eizadi P. Effect of Repetitive Feedback on Residents' Communication Skills Improvement. Acta Med Iran. 2014;52(7). [In Persian]

18. Bragard I, Merckaert I, Libert Y, Delvaux N, Etienne AM, Marchal S, et al. Communication Skills Training for Residents. Open J Med Psychol. 2012;1:68-75.

19. Suzuki LT, Mcleod H, Haufman DM, Langille DB, Sargeant J. Implementing a communication skills programme in medical schools: 
needs assessment and program change. Med Educ. 2003;36:115-124.

20. Rahimzadeh N, Karamlou Z, Malmir R, Cheshmi Zh, Hashemi A. Evaluating interpersonal communication skills among residents of Hazarate Rasoul Complex using a 360-degree approach. Razi J Med Sci. 2016;23(152). [In Persian]

21. Prasad Agrawal J. Assessment of interpersonal communication skills of intern doctors, Master of Health Profissions Education University of Maastricht the Netherlands, February 2002.

22. Ishikawa H, Son D, Eto M, Kitamura K, Kiuchi T. The informationgiving skills of resident physicians: relationships with confidence and simulated patient satisfaction. BMC Med Educ. 2017.

23. Heidarzadeh A, Dadkhah Tirani H, Asadi A, Nemati M. The Interns, Residents and Faculty Members' Knowledge and Attitudes toward Communication Skills. J Guilan Univ Med Sci. 2007;9. [In Persian]

24. Porcerelli JH, Brennan S, Carty J, Ziadni M, Markova T. Resident ratings of communication skills using the kalamazoo adapted checklist. J Grad Med Educ. 2015;7(3):458-461.

25. Khoshrang H, Salari A, Dadgaran I, Moaddab F, Rouh-Balasii L, Pourkazemi I. Quality of Education Provided at The Clinical Skills Lab From Medical Students' viewpoints in Guilan University of Medical Sciences. J Guilan Univ Med Sci. 2016;8(2):77-83. [In Persian]

26. Zamani A, Shams B, Siadat ZD, Adibi P, Salehi H, Saneii H. The Effect of Communication Skill Training on Clinical Skill of Internal Medicine and Infectious Disease Residents of Isfahan University of Medical Sciences. J Med Educ. 2006;8(2). [In Persian]

27. Nørgaard B, Ammentorp J, Ohm Kyvik K, Kofoed PE. Communication skills training increases self-efficacy of health care professionals. J Contin Educ Health Prof. 2012;32(2):90-97.

28. Alansari A. A Comparison Study of Communication Skills Between General Surgery and General Practice Residents on First-time Patient Visits. Can Med Educ J. 2012;3(1):e42-e51.

29. Bylund CL, Brown R, Gueguen JA, Diamond C, Bianculli J, Kissane DW. The implementation and assessment of a comprehensive communication skills training curriculum for oncologists. Psychooncology. 2010 Jun;19(6):583-93. 